\title{
Smooth Dynamics for Distributed Constrained Optimization with Heterogeneous Delays
}

\author{
Mengmou Li, Shunya Yamashita, Takeshi Hatanaka, and Graziano Chesi
}

\begin{abstract}
This work investigates the distributed constrained optimization problem under inter-agent communication delays from the perspective of passivity. First, we propose a continuoustime algorithm for distributed constrained optimization with general convex objective functions. The asymptotic stability under general convexity is guaranteed by the phase lead compensation. The inequality constraints are handled by adopting a projectionfree generalized Lagrangian, whose primal-dual gradient dynamics preserves passivity and smoothness, enabling the application of the LaSalle's invariance principle in the presence of delays. Then, we incorporate the scattering transformation into the proposed algorithm to enhance the robustness against unknown and heterogeneous communication delays. Finally, a numerical example of a matching problem is provided to illustrate the results.
\end{abstract}

\section{INTRODUCTION}

D ISTRIBUTED convex optimization over multi-agent systems aims to drive agents to cooperatively optimize the sum of local objective functions that are only accessible to their local agents. Ever since the pioneer work [1] that provides a control-theoretic perspective for the proportional-integral (PI) consensus-based distributed algorithms, many works have been carried out in the continuous-time scheme [2]. Recently, some problems in distributed optimization have been analyzed via passivity-based techniques [3]-[7]. Passivity-based techniques usually enjoy good scalability to large-scale networks owing to the preservation of passivity in parallel or negative feedback interconnection of passive components [8].

Distributed optimization in the presence of communication delays has been widely studied in recent years [3], [4], [9]. The work [9] addresses time-varying delays, but it only considers an identical delay known in advance for all communication channels and does not treat inequality constraints, which simplifies convergence analysis. The problem under unknown and heterogeneous communication delays is addressed via passivity techniques in [3], [4]. However, to ensure optimality in the presence of inequality constraints, delays are assumed to be homogeneous and an additional assumption on the graph is needed in [3], which is not always easy to verify in large scale networks. Besides, the objective function is assumed to be strictly convex in [3], [4], which does not hold for a large class of convex optimization problems.

M. Li and G. Chesi are with the Department of Electrical and Electronic Engineering, The University of Hong Kong, Hong Kong, China (e-mail: mengmou_li@hku.hk; chesi@eee.hku.hk).

S. Yamashita is with School of Engineering, Tokyo Institute of Technology, 2-12-1 S5-16, Ookayama, Meguro-ku, Tokyo, Japan (email:yamashita.s.ag@hfg.sc.e.titech.ac.jp).

T. Hatanaka is with Graduate School of Engineering, Osaka University, 2-1 Yamada-oka, Suita, Osaka, Japan (e-mail: hatanaka@eei.eng.osaka-u.ac.jp).
The LaSalle's invariance principle is widely used for convergence analysis of distributed algorithms. Algorithms derived from the classical Lagrange multiplier method usually adopt projected operations to guarantee the non-negativeness of the multipliers for inequality constraints. As a result, it leads to non-smooth dynamics, which is analyzed by the invariance principle for Carathéodory systems [10]. However, the discontinuous nature hinders the application of the invariance principle when delays are introduced into the systems, which results in the additional restrictive assumptions in [3], [4]. It is worth noting that a projection-free Lagrangian is adopted to solve local and couple inequalities in [11], which enables a smooth dynamics and the application of the LaSalle's invariance principle under delays. Another important issue is that the primal-dual gradient dynamics may cause oscillations when the objective function lacks strict convexity [12]. To cope with this problem, some modification methods are introduced [12], [13]. However, these methods are either restricted to affine constraints or not in a distributed structure. Recently, a phase lead compensation technique is adopted as a generalized method to ensure convergence [5].

In this work, we address unknown and heterogeneous interagent communication delays in distributed constrained optimization without the strictly convex assumption by combining techniques used in [5], [11] from the perspective of passivity. First, we propose a smooth continuous-time algorithm for distributed constrained optimization with general convex objective functions without delays. Then, we incorporate the scattering transformation into the proposed algorithm to enhance the robustness against unknown and heterogeneous communication delays.

\section{Preliminaries}

Notations: Let $\mathbb{R}\left(\mathbb{R}_{\geq 0}\right)$ be the set of (non-negative) real numbers. $\operatorname{col}\left(v_{1}, \ldots, v_{m}\right):=\left(v_{1}^{T}, \ldots, v_{m}^{T}\right)^{T}$ denotes the column vector stacked with vectors $v_{1}, \ldots, v_{m} . I_{n}$ denotes the $n \times n$ identity matrix, $\mathbf{1}_{n}:=\operatorname{col}(1, \ldots, 1) \in \mathbb{R}^{n}$, and $\mathbf{0}$ denotes the zero matrix of proper dimension. The notation "o" denotes the Hadamard product and " $\otimes$ " denotes the Kronecker product. $\nabla_{k} f$ denotes the gradient of $f$ along the variable $k$, whose subscript can be omitted if there is only one variable.

We first introduce some knowledge of convex analysis. A differentiable function $f: \mathbb{R}^{m} \rightarrow \mathbb{R}$ is convex over a convex set $\mathcal{X} \subset \mathbb{R}^{m}$ iff $[\nabla f(x)-\nabla f(y)]^{T}(x-y) \geq 0, \forall x, y \in \mathcal{X}$, and is strictly convex iff the strict inequality holds for any $x \neq y$. The function $f$ is said to be concave if $-f$ is convex. For a function $\mathcal{L}: \mathcal{X} \times \mathcal{Y} \rightarrow \mathbb{R}$ with $\mathcal{X} \subset \mathbb{R}^{n}, \mathcal{Y} \subset \mathbb{R}^{m}$ being 
closed and convex, $\left(x^{*}, y^{*}\right) \in \mathcal{X} \times \mathcal{Y}$ is called a saddle point of $\mathcal{L}$ if $\mathcal{L}\left(x^{*}, y\right) \leq \mathcal{L}\left(x^{*}, y^{*}\right) \leq \mathcal{L}\left(x, y^{*}\right), \forall(x, y) \in \mathcal{X} \times \mathcal{Y}$.

Next, let us present some basic concepts in graph theory. An undirected communication graph is represented by $\mathcal{G}=$ $(\mathcal{N}, \mathcal{E})$, where $\mathcal{N}=\{1, \ldots, N\}$ is the node set of all agents, $\mathcal{E} \subset \mathcal{N} \times \mathcal{N}$ is the edge set. The edge $(i, j) \in \mathcal{E}$ means that agent $i$ and $j$ can exchange information. The adjacency matrix $\mathcal{A}:=\left[a_{i j}\right]$ satisfies $a_{i i}=0$, and $a_{i j}=a_{j i}>0$ if $(i, j) \in \mathcal{E}$ and $a_{i j}=0$, otherwise. The graph $\mathcal{G}$ is said to be connected if there exists a sequence of successive edges between any two agents. When $\mathcal{G}$ is connected and undirected, its corresponding Laplacian matrix $L:=\operatorname{diag}\left\{\mathcal{A} \cdot \mathbf{1}_{N}\right\}-\mathcal{A}$ is positive semidefinite and has zero as its simple eigenvalue associated with eigenvector $v=\alpha \mathbf{1}_{N}, \forall \alpha \in \mathbb{R}$.

We conclude this section by giving the definition of passivity [5]. Consider a system $\Sigma$ described by a state model with state $x \in \mathbb{R}^{m}$, input $u \in \mathbb{R}^{n}$ and output $y \in \mathbb{R}^{n}$. The system $\Sigma$ is said to be passive if there exists a positive semidefinite differentiable function $S(x): \mathbb{R}^{m} \rightarrow \mathbb{R}_{>0}$ called storage function, such that $\dot{S}(x) \leq y^{T} u$ holds for all inputs $u(t)$, all initial states $x(0)$, and all $t \geq 0$.

\section{PASSIVITy-BASED Algorithm On CONSTRAined DISTRIBUTED OPTIMIZATION}

Let us consider a constrained distributed optimization problem in a network of $N$ agents in the node set $\mathcal{N}=\{1, \ldots, N\}$

$$
\min _{z} \sum_{i \in \mathcal{N}} f_{i}(z), \text { s.t. } g_{i}(z) \leq 0, h_{i}(z)=0, i \in \mathcal{N}
$$

where $z \in \mathbb{R}^{n}$ is a decision variable, $f_{i}: \mathbb{R}^{n} \rightarrow \mathbb{R}$, $g_{i}: \mathbb{R}^{n} \rightarrow \mathbb{R}, h_{i}: \mathbb{R}^{n} \rightarrow \mathbb{R}$ are local objective function, inequality constraint and affine equality constraint for the $i$ th agent, respectively. Just for simplicity, we only consider one local inequality and equality constraint for each agent, while it is trivial to extend subsequent results to the case with multiple local constraints. Next, we adopt the following assumptions.

Assumption 1. The functions $f_{i}$ and $g_{i}$ are convex and twice differentiable. The Slater's condition holds and there exists a finite optimal solution to problem (1).

This assumption ensures that the problem is well-defined. $f_{i}$ is only required to be convex, implying that there may exist more than one optimal solution to problem (1).

Assumption 2. The communication graph $\mathcal{G}$ is undirected and connected.

Denote $x=\operatorname{col}\left(x_{1}, \ldots, x_{N}\right)$, where $x_{i} \in \mathbb{R}^{n}$, then problem (1) is equivalent to

$$
\begin{aligned}
& \min _{x} f(x):=\sum_{i \in \mathcal{N}} f_{i}\left(x_{i}\right)+x^{T} \mathbf{L} x \\
& \text { s.t. } g_{i}\left(x_{i}\right) \leq 0, h_{i}\left(x_{i}\right)=0, i \in \mathcal{N}, \mathbf{L} x=\mathbf{0}
\end{aligned}
$$

where $\mathbf{L}=L \otimes I_{n}$, and $L$ is the Laplacian matrix.

\section{A. Generalized Lagrange Multiplier Method}

In this subsection, we briefly review the generalized Lagrange multiplier method (GLMM) in [11] for solving problem
(2). Define compact variables $\lambda=\operatorname{col}\left(\lambda_{1}, \ldots, \lambda_{N}\right), \mu=$ $\operatorname{col}\left(\mu_{1}, \ldots, \mu_{N}\right), \xi=\operatorname{col}\left(\xi_{1}, \ldots, \xi_{N}\right)$ with $\lambda_{i}, \mu_{i} \in \mathbb{R}$ and $\xi_{i} \in \mathbb{R}^{n}, i \in \mathcal{N}$. Adopt a Lagrangian for problem (2),

$$
\begin{aligned}
& \mathcal{L}(x, \xi, \lambda, \mu):= \\
& f(x)+\sum_{i \in \mathcal{N}} \lambda_{i}^{2} g_{i}\left(x_{i}\right)+\sum_{i \in \mathcal{N}} \mu_{i} h_{i}\left(x_{i}\right)-\xi^{T} \mathbf{L} x+\frac{1}{2} x^{T} \mathbf{L} x
\end{aligned}
$$

where $\mathcal{L}(x, \xi, \lambda, \mu)$ is a class of the generalized Lagrangian; $\lambda_{i}^{2}$ is the generalized multiplier for the inequality constraint $g_{i} \leq 0 ; \mu_{i}$ is the multiplier for the equality constraint $h_{i}=0$; $\xi$ is the multiplier for the consensus constraint $\mathbf{L} x=\mathbf{0}$. Then, by applying the primal-dual gradient flow to $\mathcal{L}(x, \xi, \lambda, \mu)$, we obtain the following projection-free distributed algorithm

$$
\begin{aligned}
\dot{x}_{i}= & -\nabla_{x_{i}} \mathcal{L}=-\nabla f_{i}\left(x_{i}\right)-\lambda_{i}^{2} \nabla g_{i}\left(x_{i}\right)-\mu_{i} \nabla h_{i}\left(x_{i}\right) \\
& +\sum_{j \in \mathcal{N}_{i}} a_{i j}\left(x_{j}-x_{i}\right)-\sum_{j \in \mathcal{N}_{i}} a_{i j}\left(\xi_{j}-\xi_{i}\right) \\
\dot{\xi}_{i}= & \nabla_{\xi_{i}} \mathcal{L}, \dot{\lambda}_{i}=\nabla_{\lambda_{i}} \mathcal{L}, \dot{\mu}_{i}=\nabla_{\mu_{i}} \mathcal{L}
\end{aligned}
$$

where $a_{i j}$ is the $(i, j)$-th entry of the adjacency matrix. The above algorithm is said to be projection-free since the nonnegativeness of multipliers $\lambda_{i}^{2}$ is already guaranteed without any projection operator.

Let $\left(x^{*}, \xi^{*}, \lambda^{*}, \mu^{*}\right) \in \mathcal{H}^{*}$ denotes an optimal solution of the problem where $\mathcal{H}^{*}$ is the set satisfying the generalized KKT condition for problem (2) corresponding to $\mathcal{L}(x, \xi, \lambda, \mu)$, i.e.,

$\mathbf{L} x^{*}=\mathbf{0}$,

$h_{i}\left(x_{i}^{*}\right)=0, \quad g_{i}\left(x_{i}^{*}\right) \leq 0, \quad \lambda_{i}^{* 2} g_{i}\left(x_{i}^{*}\right)=0$,

$\nabla f_{i}\left(x_{i}^{*}\right)+\lambda_{i}^{* 2} \nabla g_{i}\left(x_{i}^{*}\right)+\mu_{i}^{*} \nabla h_{i}\left(x_{i}^{*}\right)+\sum_{j=1}^{N} a_{i j}\left(\xi_{j}^{*}-\xi_{i}^{*}\right)=\mathbf{0}$

where the term $\sum_{j \in \mathcal{N}_{i}} a_{i j}\left(x_{j}^{*}-x_{i}^{*}\right)$ in 5c is omitted since (5a) implies $x_{i}^{*}=x_{j}^{*}, \forall i, j$. Next, let us give the following lemma derived from [11].

Lemma 1. Under Assumption 1, a fixed point $\left(x^{*}, \xi^{*}, \lambda^{*}, \mu^{*}\right)$ solves problem (2) if and only if it satisfies condition (5).

Then, denote $z^{*}=x_{i}^{*}, \forall i$. Obviously, $z^{*}$ is the optimal solution to problem (1).

\section{B. GLMM With Phase Lead Compensation}

A restriction for the convergence of algorithm (4) is $f_{i}$ being strictly convex [11]. When $f_{i}$ lacks strict convexity, an extra modification is needed. In this subsection, we add the phase lead compensator into the dynamics (4), which serves to provide stable zeros and avoid possible oscillations for the algorithm under general convexity [5].

Define $\nu=\operatorname{col}\left(\nu_{1}, \ldots, \nu_{N}\right)$ with $\nu_{i} \in \mathbb{R}^{n}$ and

$$
\begin{aligned}
\nu_{i}= & -\nabla f_{i}\left(x_{i}\right)-\lambda_{i}^{2} \nabla g_{i}\left(x_{i}\right)-\mu_{i} \nabla h_{i}\left(x_{i}\right) \\
& +\sum_{j \in \mathcal{N}_{i}} a_{i j}\left(x_{j}-x_{i}\right)-\sum_{j \in \mathcal{N}_{i}} a_{i j}\left(\xi_{j}-\xi_{i}\right) .
\end{aligned}
$$

We add the phase lead compensator to the integrator in the primal gradient dynamics (4a), then the dynamics for the $i$ th agent is reformulated in the frequency domain as

$$
x_{i}(s)=\left(M_{i}(s) \cdot I_{n}\right) \nu_{i}(s)
$$




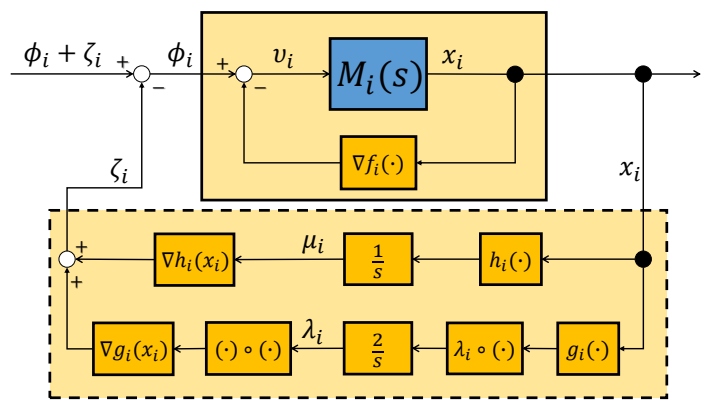

Fig. 1. Block diagram of the $i$ th agent's dynamics for $x_{i}, \lambda_{i}, \nu_{i}$. The generalized transfer function $M_{i}(s)$ represents the cascade connection of phase lead compensator with the integrator for gradient dynamics of $x_{i}$ to ensure convergence under general convexity.

where the generalized transfer function $M_{i}(s)$ is defined by

$$
\begin{aligned}
& M_{i}(s)=\sum_{k=1}^{m} \frac{c_{k}^{i}}{s+b_{k}^{i}}, \\
& b_{m}^{i}>\ldots>b_{2}^{i}>b_{1}^{i}=0, c_{k}^{i}>0, k=1, \ldots, m \geq 2 .
\end{aligned}
$$

Note that we only apply the phase lead compensator to $4 \mathrm{a}$, and (8) is a simplified version of the algorithm in [5].

Then, the overall distributed algorithm becomes

$$
\begin{aligned}
\dot{\rho}_{k}^{i}= & -b_{k}^{i} \rho_{k}^{i}+c_{k}^{i} \nu_{i}, k=1, \ldots, m \\
x_{i}= & \sum_{k=1}^{m} \rho_{k}^{i} \\
\nu_{i}= & -\nabla f_{i}\left(x_{i}\right)-\lambda_{i}^{2} \nabla g_{i}\left(x_{i}\right)-\mu_{i} \nabla h_{i}\left(x_{i}\right) \\
& +\sum_{j \in \mathcal{N}_{i}} a_{i j}\left(x_{j}-x_{i}\right)-\sum_{j \in \mathcal{N}_{i}} a_{i j}\left(\xi_{j}-\xi_{i}\right) \\
\dot{\xi}_{i}= & \sum_{j \in \mathcal{N}_{i}} a_{i j}\left(x_{j}-x_{i}\right) \\
\dot{\lambda}_{i}= & 2 \lambda_{i} g_{i}\left(x_{i}\right), \dot{\mu}_{i}=h_{i}\left(x_{i}\right)
\end{aligned}
$$

where $9 \mathrm{a}, 9 \mathrm{~b}$ ) is the state-space representation of (7) and $\rho_{k}^{i} \in \mathbb{R}^{n}(k=1, \ldots, m)$ is an auxiliary state variable. Under the phase lead compensation, the block diagram of the $i$ th agent's dynamics for $x_{i}, \lambda_{i}, \nu_{i}$ can be described by Figure 1 . We can observe that, algorithm (9) is reduced to algorithm (4) if $M_{i}(s)$ is replaced by an integrator.

\section{Convergence Analysis}

We aim to address convergence of system (9) via passivity analysis in this subsection. To this end, let us first analyze the passivity of the subsystems included in it. Denote a fixed point $\left(x^{*}, \xi^{*}, \lambda^{*}, \mu^{*}\right) \in \mathcal{H}^{*}$ as the reference point, $\nu=\operatorname{col}\left(\nu_{1}, \ldots, \nu_{N}\right)$ and $\nu^{*}=\nu\left(x^{*}, \xi^{*}, \lambda^{*}, \mu^{*}\right)=0$. First, we focus on subsystem $9 \mathrm{a}$ - $-(9 \mathrm{c}$ and obtain the following lemma whose proof can be found in [5, Lemma 7].

Lemma 2 ( [5]). Under Assumption 1] the system defined by (9a)-(9c) is passive from $\phi_{i}-\phi_{i}^{*}$ to $x_{i}-z^{*}$ with respect to the storage function $S_{i}^{c}=\frac{1}{2 c_{1}^{i}}\left\|\rho_{1}^{i}-z^{*}\right\|^{2}+\sum_{k=2}^{m} \frac{1}{2 c_{k}^{i}}\left\|\rho_{k}^{i}\right\|^{2}$, where $\phi_{i}:=\nu_{i}+\nabla f_{i}\left(x_{i}\right)$ and $\phi_{i}^{*}:=\nu_{i}^{*}+\nabla f_{i}\left(x_{i}^{*}\right)$.
Briefly note that it satisfies that

$$
\dot{S}_{i}^{c}=\left(x_{i}-z^{*}\right)^{T} \nu_{i}-\sum_{k=2}^{m} \frac{b_{k}^{i}}{c_{k}^{i}} \rho_{k}^{i^{T}} \rho_{k}^{i} \leq\left(x_{i}-z^{*}\right)^{T}\left(\phi_{i}-\phi_{i}^{*}\right) .
$$

Next, we show that the dual gradient of Lagrangian (3) with respect to $\lambda, \mu$ preserves passivity.

Lemma 3. Under Assumption [1] the system given by 9e is passive from $x_{i}-z^{*}$ to $\zeta_{i}-\zeta_{i}^{*}$ with respect to the storage function $S_{i}^{g}=\frac{1}{4}\left(\lambda_{i}^{2}-\lambda_{i}^{* 2}\right)-\frac{1}{2} \lambda_{i}^{* 2}\left(\ln \lambda_{i}-\ln \lambda_{i}^{*}\right)+$ $\frac{1}{2}\left(\mu_{i}-\mu_{i}^{*}\right)^{2}$ where $\lambda_{i} \ln \lambda_{i}$ is defined as 0 when $\lambda_{i}=0$, $\zeta_{i}:=\lambda_{i}^{2} \nabla g_{i}\left(x_{i}\right)+\mu_{i} \nabla h_{i}\left(x_{i}\right)$, and $\zeta_{i}^{*}:=\zeta_{i}\left(z^{*}, \lambda_{i}^{*}, \mu_{i}^{*}\right)$.

Proof. The storage function $S_{i}^{g}$ is smooth and differentiable for $\lambda_{i}$ on $[0,+\infty]$. By direct calculation, $S_{i}^{g} \geq 0$ and $S_{i}^{g}=0$ if and only if $\left(\lambda_{i}, \mu_{i}\right)=\left(\lambda_{i}^{*}, \mu_{i}^{*}\right)$. The time derivative of $S_{i}^{g}$ gives

$$
\begin{aligned}
\dot{S}_{i}^{g}= & \left(\lambda_{i}^{2}-\lambda_{i}^{* 2}\right) g_{i}\left(x_{i}\right)+\left(\mu_{i}-\mu_{i}^{*}\right) h_{i}\left(x_{i}\right) \\
\leq & \lambda_{i}^{2}\left[g_{i}\left(z^{*}\right)+\nabla g_{i}\left(x_{i}\right)^{T}\left(x_{i}-z^{*}\right)\right] \\
& -\lambda_{i}^{* 2}\left[g_{i}\left(z^{*}\right)+\nabla g_{i}\left(z^{*}\right)^{T}\left(x_{i}-z^{*}\right)\right] \\
& +\left(\mu_{i}-\mu_{i}^{*}\right)\left[\nabla h_{i}\left(x_{i}\right)^{T}\left(x_{i}-z^{*}\right)\right] \\
= & {\left[\lambda_{i}^{2} \nabla g_{i}\left(x_{i}\right)-\lambda_{i}^{* 2} \nabla g_{i}\left(z^{*}\right)\right]^{T}\left(x_{i}-z^{*}\right)+\lambda_{i}^{2} g_{i}\left(z^{*}\right) } \\
& -\lambda_{i}^{* 2} g_{i}\left(z^{*}\right)+\left(\mu_{i}-\mu_{i}^{*}\right)\left[\nabla h_{i}\left(x_{i}\right)^{T}\left(x_{i}-z^{*}\right)\right] \\
\leq & \left(\zeta_{i}-\zeta_{i}^{*}\right)^{T}\left(x_{i}-z^{*}\right),
\end{aligned}
$$

where the first inequality follows from the convexity of $g_{i}$ and affine properties of $h_{i}$, the second inequality follows from $\nabla h_{i}\left(x_{i}\right)=\nabla h_{i}\left(z^{*}\right)$ and the KKT condition (5b).

We can also observe from Figure 1 that, the system enclosed by the solid line is passive from $\phi_{i}-\phi_{i}^{*}$ to $x_{i}-z^{*}$ by Lemma 2 The system within the dashed line is passive from $x_{i}-z^{*}$ to $\zeta_{i}-\zeta_{i}^{*}$ by Lemma 3 Moreover, since the communication part in 9c], 9d) inherits passivity [3], the overall system (9) can be seen as a feedback interconnection of passive systems. Then, we can obtain the following result on convergence.

Theorem 1. Under Assumptions 1 and 2 the trajectories of system (9) with initial condition $\lambda_{i}(0)>0, \forall i \in \mathcal{N}$ will converge to a fixed equilibrium point that solves problem (2).

Proof. Adopt the Lyapunov function candidate $V=$ $\sum_{i \in \mathcal{N}} S_{i}^{g}+\sum_{i \in \mathcal{N}} S_{i}^{c}+\frac{1}{2}\left\|\xi-\xi^{*}\right\|^{2} \geq 0$. Apparently, $V$ is radially unbounded. Denote $\zeta=\operatorname{col}\left(\zeta_{1}, \ldots, \zeta_{N}\right), \zeta^{*}=$ $\operatorname{col}\left(\zeta_{1}^{*}, \ldots, \zeta_{N}^{*}\right)$, by Lemmas 2 and 3 , the time derivative of $V$ satisfies

$$
\begin{aligned}
\dot{V} \leq & \left(x-x^{*}\right)^{T}\left(\phi-\phi^{*}\right)+\left(\zeta-\zeta^{*}\right)^{T}\left(x-x^{*}\right) \\
& -\left(\xi-\xi^{*}\right)^{T} \mathbf{L}\left(x-x^{*}\right) \leq-\left(x-x^{*}\right)^{T} \mathbf{L}\left(x-x^{*}\right) \leq 0,
\end{aligned}
$$

where the last inequality follows from the positive semidefiniteness of $\mathbf{L}$. Then the states are bounded. The set $\Omega_{0}:=$ $\{(x, \xi, \lambda, \mu, \rho) \mid V \leq V(0)\}$ is a positively invariant set. Invoking the LaSalle's invariance principle, the states will converge to the largest invariant set in $\{(x, \xi, \lambda, \mu, \rho) \mid \dot{V}=0\}$ which we denote as $\Omega_{c}$ in the subsequent, and $\dot{V}=0$ only if all the nonnegative terms are zero. Notice that $-\sum_{k=2}^{m} \frac{b_{k}^{i}}{c_{k}^{i}} \rho_{k}^{i} \rho_{k}^{T}=0$ holds only if $\rho_{k}^{i} \equiv \mathbf{0}, \forall k=2, \ldots, m$, which implies that $\nu_{i}=\mathbf{0}$ and thus $x_{i}$ is unchanged. $-\left(x-x^{*}\right)^{T} \mathbf{L}\left(x-x^{*}\right)=0$ implies that $\mathbf{L} x=\mathbf{0}$ and $\dot{\xi}=\mathbf{0}$. Then $x$ satisfies 5c]. 
Next, let us look at the dynamics $9 \mathrm{e}$ when the states converges to $\Omega_{c}$. If $\lambda_{i}^{*}=0$, then the constraint $g_{i}\left(x_{i}\right)$ is inactive, meaning that $g_{i}\left(x_{i}\right) \leq 0$ when the primal gradient of $\mathcal{L}$ with respect to $x_{i}$ vanishes, i.e., when $5 \mathrm{c}$ holds. If $\lambda_{i}^{*}>0$, recalling the definition of $S_{i}^{g}$, we obtain that $\lambda_{i}>0$, for all $t>0$, because if $\lambda_{i} \rightarrow 0$, then $S_{i}^{g} \rightarrow+\infty$ due to the term $-\frac{1}{2} \lambda_{i}^{* 2} \ln \lambda_{i}$, which contradicts the fact that $V$ is decreasing. Since $\lambda_{i}$ is nonzero, $g_{i}\left(x_{i}\right)$ should be zero to ensure stability and boundedness of the dynamics $\dot{\lambda}_{i}=2 \lambda_{i} g_{i}$. Similarly, it can be observed that $h_{i}\left(x_{i}\right)$ should be zero to ensure boundedness. Therefore, the KKT condition (5) is satisfied, i.e., $(x, \xi, \lambda, \mu) \in \mathcal{H}^{*}$ and is unchanged whenever $(x, \xi, \lambda, \mu, \rho)$ converges to $\Omega_{c}$. In conclusion, the trajectories generated by the algorithm will asymptotically converge to a constant equilibrium point that solves problem (2).

\section{Constrained Distributed Optimization with Heterogeneous Communication Delays}

Let us consider the presence of unknown and heterogeneous inter-agent communication delays in this section. For $(i, j) \in$ $\mathcal{E}$, let the communication delay from agent $i$ to $j$ be denoted by a constant $T_{i j}$. In this case, each agent cannot catch the current variable of its neighboring agents. Then the algorithm under delays becomes

$$
\begin{aligned}
\nu_{i}= & -\nabla f_{i}\left(x_{i}\right)-\lambda_{i}^{2} \nabla g_{i}\left(x_{i}\right)-\mu_{i} \nabla h_{i}\left(x_{i}\right) \\
& +\sum_{j \in \mathcal{N}_{i}} a_{i j}\left(r_{i j}^{x}-x_{i}\right)-\sum_{j \in \mathcal{N}_{i}} a_{i j}\left(r_{i j}^{\xi}-\xi_{i}\right) \\
\dot{\xi}_{i}= & \sum_{j \in \mathcal{N}_{i}} a_{i j}\left(r_{i j}^{x}-x_{i}\right)
\end{aligned}
$$

$$
\text { 9a), 9b, 9e }
$$

where (11a) and $(11 \mathrm{~b})$ are the modified update expression against $\overline{(9 \mathrm{c})}$ and $(9 \mathrm{~d})$ by replacing the neighbor's information with $r_{i j}^{x}, r_{i j}^{\xi}$, which denote the information agent $i$ receives from agent $j$. The dynamics (11a), (11b) can be rewritten as

$$
\left[\begin{array}{c}
\nu_{i} \\
\dot{\xi}_{i}
\end{array}\right]=\left[\begin{array}{c}
-\nabla f_{i}\left(x_{i}\right)-\zeta_{i} \\
\mathbf{0}
\end{array}\right]+p_{i}
$$

where $\zeta_{i}$ is defined in Lemma 3, $p_{i}:=\sum_{j \in \mathcal{N}_{i}} p_{i j}, p_{i j}:=$ $\left[\begin{array}{l}p_{i j}^{x} \\ p_{i j}^{\xi}\end{array}\right]=E_{i j}\left[\begin{array}{l}r_{i j}^{x}-x_{i} \\ r_{i j}^{\xi}-\xi_{i}\end{array}\right]$ and $E_{i j}:=\left[\begin{array}{cc}a_{i j} & -a_{i j} \\ a_{i j} & 0\end{array}\right] \otimes I_{n}, j \in$

Define $\bar{x}_{i}:=x_{i}-z^{*}, \bar{\xi}_{i}:=\xi_{i}-2 \xi_{i}^{*}, \bar{p}_{i}:=p_{i}-\sum_{j \in \mathcal{N}_{i}} p_{i j}^{*}=$ $\sum_{j \in \mathcal{N}_{i}} \bar{p}_{i j}, \quad p_{i j}^{*}:=a_{i j}\left[\begin{array}{c}\xi_{i}^{*}-\xi_{j}^{*} \\ \mathbf{0}\end{array}\right], r_{i j}:=\left[\begin{array}{c}r_{i j}^{x} \\ r_{i j}^{\xi}\end{array}\right], \quad \bar{r}_{i j}:=$ $\left[\begin{array}{c}\bar{r}_{i j}^{x} \\ \bar{r}_{i j}^{\xi}\end{array}\right]=r_{i j}-r_{i j}^{*}, \quad r_{i j}^{*}:=\left[\begin{array}{c}z^{*} \\ \xi_{i}^{*}-\xi_{j}^{*}\end{array}\right]$. We show that the dynamics under communication delays preserves passivity-like properties.

Lemma 4. Under Assumption 1] the system given by (11) has the passivity-like property $\dot{S}_{i} \leq \sum_{j \in \mathcal{N}_{i}} \bar{r}_{i j}^{T} \bar{p}_{i j}$ where $S_{i}=$ $S_{i}^{c}+S_{i}^{g}+\frac{1}{2}\left\|\bar{\xi}_{i}\right\|^{2}, S_{i}^{c}$ and $S_{i}^{g}$ are defined in Lemmas 2 and 3
Proof. From the former lemmas, $S_{i} \geq 0$. The time derivative of $S_{i}$ satisfies

$$
\begin{aligned}
& \dot{S}_{i} \leq-\sum_{k=2}^{m} \frac{b_{k}^{i}}{c_{k}^{i}} \rho_{k}^{i} \rho_{k}^{T}+\bar{x}_{i}^{T}\left[-\nabla f_{i}\left(x_{i}\right)-\zeta_{i}\right] \\
& +\bar{x}_{i}^{T}\left(\zeta_{i}-\zeta_{i}^{*}\right)+\left[\begin{array}{ll}
\bar{x}_{i} & \bar{\xi}_{i}
\end{array}\right] p_{i} \\
& \leq-\bar{x}_{i}^{T}\left(\zeta_{i}-\zeta_{i}^{*}\right)+\bar{x}_{i}^{T}\left(\zeta_{i}-\zeta_{i}^{*}\right)-\sum_{k=2}^{m} \frac{b_{k}^{i}}{c_{k}^{i}} \rho_{k}^{i} \rho_{k}^{i} \\
& +\bar{x}_{i}^{T} \sum_{j \in \mathcal{N}_{i}} a_{i j}\left(\xi_{j}^{*}-\xi_{i}^{*}\right)+\sum_{j \in \mathcal{N}_{i}}\left[\bar{x}_{i} \bar{\xi}_{i}\right] p_{i j} \\
& =-\sum_{k=2}^{m} \frac{b_{k}^{i}}{c_{k}^{i}} \rho_{k}^{i T} \rho_{k}^{i}+\sum_{j \in \mathcal{N}_{i}}\left[\begin{array}{c}
\bar{x}_{i}+\bar{r}_{i j}^{x}-\bar{r}_{i j}^{x} \\
\bar{\xi}_{i}+\bar{r}_{i j}^{\xi}-\bar{r}_{i j}^{\xi}
\end{array}\right]^{T} \bar{p}_{i j} \\
& \begin{array}{l}
\leq \sum_{j \in \mathcal{N}_{i}} \bar{r}_{i j}^{T} \bar{p}_{i j}-\sum_{j \in \mathcal{N}_{i}} a_{i j}\left\|\bar{x}-\bar{r}_{i j}^{x}\right\|^{2} \\
\leq \sum_{j \in \mathcal{N}_{i}} \bar{r}_{i j}^{T} \bar{p}_{i j}
\end{array}
\end{aligned}
$$

where the first inequality follows from $[10, \sqrt{12}$ and Lemma 3

If state variables are exchanged, then agent $i$ at time $t$ receives $r_{i j}^{x}=x_{j}\left(t-T_{j i}\right)$ and $r_{i j}^{\xi}=\xi_{j}\left(t-T_{j i}\right)$ from agent $j$ due to the existence of delays. However, this may cause instability and divergence to the dynamics [3]. Thus, we do not directly exchange original state variables here. To ensure stability under delays, a scattering transformation method is introduced [8], [14]. The scattering transformation in this work is defined as

$$
\begin{aligned}
& s_{\overrightarrow{i j}}=\frac{1}{\sqrt{2 \eta}}\left(-p_{i j}+\eta r_{i j}\right), \quad s_{i j}=\frac{1}{\sqrt{2 \eta}}\left(p_{i j}+\eta r_{i j}\right) \\
& s_{\overleftarrow{j i}}=\frac{1}{\sqrt{2 \eta}}\left(p_{j i}+\eta r_{j i}\right), \quad s_{\overrightarrow{j i}}=\frac{1}{\sqrt{2 \eta}}\left(-p_{j i}+\eta r_{j i}\right)
\end{aligned}
$$

for $(i, j) \in \mathcal{E}$, where $\eta>0$. Specifically, $s \overrightarrow{i j}$ denotes the signal that agent $i$ sends to agent $j$ while $s \overleftarrow{j i}$ represents the signal $j$ receives from $i$. The other notations are defined similarly. Due to the delays, these signals should satisfy

$$
s_{\overleftarrow{j i}}(t)=s_{\overrightarrow{i j}}\left(t-T_{i j}\right), \quad s \overleftarrow{i j}(t)=s_{\overrightarrow{j i}}\left(t-T_{j i}\right)
$$

Instead of directly exchanging $x$ and $\xi$, scattering variables (14) are exchanged between agent $i$ and $j$ for $(i, j) \in \mathcal{E}$. Then the input variables $r_{i j}^{x}, r_{i j}^{\xi}$ for each agent are computed from these scattering variables. For simplicity, we suppose that $s \overleftarrow{i j}(t)=s_{\overrightarrow{i j}}(t)=0, \forall t<0$

It has been proved that the system of the scattering transformation inherits passivity properties.

Lemma 5 ( [3]). The system consisting of (14] and (15p) is passive from $-\left[\bar{p}_{i j}^{T}, \bar{p}_{j i}^{T}\right]^{T}$ to $\left[\bar{r}_{i j}^{T}, \bar{r}_{j i}^{T}\right]^{T}$ with respect to the storage function

$$
\begin{aligned}
V_{i j}(t)=\frac{1}{2} \int_{0}^{t} & \left(\left\|s \overrightarrow{i j}(\tau)+\gamma_{i j}^{*}\right\|^{2}-\left\|s \overleftarrow{j i}(\tau)+\gamma_{i j}^{*}\right\|^{2}\right. \\
& \left.+\left\|s \overrightarrow{j i}(\tau)-\delta_{i j}^{*}\right\|^{2}-\left\|s \overleftarrow{i j}(\tau)-\delta_{i j}^{*}\right\|^{2}\right) d \tau \\
& +\frac{T_{i j}}{2}\left(\gamma_{i j}^{*}\right)^{2}+\frac{T_{j i}}{2}\left(\delta_{i j}^{*}\right)^{2}
\end{aligned}
$$

where $\gamma_{i j}^{*}:=\frac{1}{\sqrt{2 \eta}}\left(p_{i j}^{*}-\eta r_{i j}^{*}\right), \delta_{i j}^{*}:=\frac{1}{\sqrt{2 \eta}}\left(p_{i j}^{*}+\eta r_{i j}^{*}\right)$.

Since $\bar{p}_{i j}=p_{i j}-p_{i j}^{*}$, we can easily obtain the following lemma by taking $p_{i j}^{*}=p_{j i}^{*}=\mathbf{0}$ in the proof of Lemma 5

Lemma 6. The system consisting of (14) and (15) is passive from $-\left[p_{i j}^{T}, p_{j i}^{T}\right]^{T}$ to $\left[\bar{r}_{i j}^{T}, \bar{r}_{j i}^{T}\right]^{T}$. 
Following the above lemmas, the algorithm with scattering transformation controllers can be viewed as a feedback interconnection of passive systems and hence preserves passivity. Then, we can obtain the convergence result.

Theorem 2. Under Assumptions 1 and 2, the trajectories of system (11) with controller (14), (15) and initial condition $\lambda_{i}(0)>0, \forall i \in \mathcal{N}$ will asymptotically converge to a fixed equilibrium that solves problem (2).

Proof. Step 1: We adopt the Lyapunov function candidate $\bar{V}=\sum_{i \in \mathcal{N}} S_{i}+\sum_{(i, j) \in \mathcal{E}} V_{i j}$ where $S_{i}$ and $V_{i j}$ are defined in Lemma 4 and Lemma 5 , respectively. Obviously, $\bar{V} \geq 0$ and is radially unbounded. By following Lemma 4 and Lemma 5 . the time derivative of $\bar{V}$ satisfies

$$
\dot{\bar{V}} \leq \sum_{i \in \mathcal{N}}\left\{-\sum_{k=2}^{m} \frac{b_{k}^{i}}{c_{k}^{i}} \rho_{k}^{i T} \rho_{k}^{i}-\sum_{j \in \mathcal{N}_{i}} a_{i j}\left\|\bar{x}-\bar{r}_{i j}^{x}\right\|^{2}\right\} \leq 0
$$

Then the system states are bounded. The set $\bar{\Omega}_{0}:=$ $\{(x, \xi, \lambda, \mu, \rho) \mid \bar{V} \leq \bar{V}(0)\}$ is a positively invariant set. By the LaSalle's invariance principle for delay systems [15, Theorem 5.17], the states will converge to the largest invariant set $\bar{\Omega}_{c}$ in $\{(x, \xi, \lambda, \mu, \rho) \mid \dot{\bar{V}}=0\}$, which implies that $\dot{\xi}_{i}=\mathbf{0}, x_{i}=r_{i j}^{x}$. Moreover, $\nu_{i}=\mathbf{0}$ implies that $x_{i}$ remains unchanged.

However, these results derived from (16) are insufficient to conclude the optimality yet. To this end, let us go back and rearrange the time derivative of $\dot{\bar{V}}$.

Step 2: Reformulating the term $\sum_{j \in \mathcal{N}_{i}}\left[\begin{array}{ll}\bar{x}_{i} & \bar{\xi}_{i}\end{array}\right] p_{i j}$ from (13), we have

$$
\begin{aligned}
& \sum_{j \in \mathcal{N}_{i}}\left[\bar{x}_{i} \bar{\xi}_{i}\right] p_{i j} \\
& =\sum_{j \in \mathcal{N}_{i}}\left(\left[\begin{array}{cc}
\bar{r}_{i j}^{x} & \bar{r}_{i j}^{\xi}
\end{array}\right]-\left[\begin{array}{cc}
\bar{r}_{i j}^{x}-\bar{x}_{i} & \bar{r}_{i j}^{\xi}-\bar{\xi}_{i}
\end{array}\right]\right) p_{i j} \\
& =\sum_{j \in \mathcal{N}_{i}} \bar{r}_{i j}^{T} p_{i j}-\sum_{j \in \mathcal{N}_{i}}\left[\begin{array}{c}
r_{i j}^{x}-x_{i} \\
r_{i j}^{\xi}-\xi_{i}
\end{array}\right]^{T} E_{i j}\left[\begin{array}{c}
r_{i j}^{x}-x_{i} \\
r_{i j}^{\xi}-\xi_{i}
\end{array}\right] \\
& -\sum_{j \in \mathcal{N}_{i}}\left[\begin{array}{c}
\mathbf{0} \\
\xi_{i}^{*}-\xi_{j}^{*}
\end{array}\right]^{T} E_{i j}\left[\begin{array}{c}
r_{i j}^{x}-x_{i} \\
r_{i j}^{\xi}-\xi_{i}
\end{array}\right] \\
& =\sum_{j \in \mathcal{N}_{i}} \bar{r}_{i j}^{T} p_{i j}-\sum_{j \in \mathcal{N}_{i}} a_{i j}\left(\xi_{i}^{*}-\xi_{j}^{*}\right)^{T}\left(r_{i j}^{x}-x_{i}\right) \\
& -\sum_{j \in \mathcal{N}_{i}} a_{i j}\left\|x_{i}-r_{i j}^{x}\right\|^{2} \text {. }
\end{aligned}
$$

When the states are in $\bar{\Omega}_{c}$, it is already shown that $x_{i}=r_{i j}^{x}=$ constant. Thus, following the time derivative of $\bar{V}$ along with Lemma 6, we have

$$
\begin{aligned}
\dot{\bar{V}}= & \sum_{i \in \mathcal{N}}\left\{-\sum_{k=2} m \rho_{k}^{i} \rho_{k}^{i}+\left(\mu_{i}-\mu_{i}^{*}\right) h_{i}\left(x_{i}\right)\right. \\
& +\left(\lambda_{i}^{2}-\lambda_{i}^{* 2}\right) g_{i}\left(x_{i}\right)-\bar{x}_{i}^{T}\left[\nabla f_{i}\left(x_{i}\right)+\lambda_{i}^{2} \nabla g_{i}\left(x_{i}\right)\right] \\
& \left.-\sum_{j \in \mathcal{N}_{i}} a_{i j}\left\|x_{i}-r_{i j}^{x}\right\|^{2}\right\} \\
= & \sum_{i \in \mathcal{N}}\left\{-\bar{x}_{i}^{T}\left[\nabla f_{i}\left(x_{i}\right)+\lambda_{i}^{2} \nabla g_{i}\left(x_{i}\right)\right]+\left(\lambda_{i}^{2}-\lambda_{i}^{* 2}\right) g_{i}\left(x_{i}\right)\right. \\
& \left.+\left(\mu_{i}-\mu_{i}^{*}\right) h_{i}\left(x_{i}\right)\right\} \\
= & -\bar{x}^{T} \nabla_{x} \mathcal{L}_{g}+\left(\lambda^{2}-\lambda^{* 2}\right)^{T} \nabla_{\lambda^{2}} \mathcal{L}_{g}+\bar{\mu}^{T} \nabla_{\mu} \mathcal{L}_{g} \\
\leq & \mathcal{L}_{g}\left(x^{*}, \lambda, \mu\right)-\mathcal{L}_{g}(x, \lambda, \mu)+\mathcal{L}_{g}(x, \lambda, \mu)-\mathcal{L}_{g}\left(x, \lambda^{*}, \mu^{*}\right)
\end{aligned}
$$

$$
\begin{aligned}
= & \mathcal{L}_{g}\left(x^{*}, \lambda, \mu\right)-\mathcal{L}_{g}\left(x^{*}, \lambda^{*}, \mu^{*}\right) \\
& +\mathcal{L}_{g}\left(x^{*}, \lambda^{*}, \mu^{*}\right)-\mathcal{L}_{g}\left(x, \lambda^{*}, \mu^{*}\right)
\end{aligned}
$$

where $\lambda^{2}:=\lambda \circ \lambda$ and $\lambda^{* 2}$ is defined similarly, $\mathcal{L}_{g}(x, \lambda, \mu):=$ $f(x)+\sum_{i \in \mathcal{N}}\left[\lambda_{i}^{2} g_{i}\left(x_{i}\right)+\mu_{i} h_{i}\left(x_{i}\right)\right]$ is a Lagrangian, the third equality follows from the fact that $\mathcal{L}_{g}$ is convex with respect to $x$ and concave with respect to $\left(\lambda^{2}, \mu\right)$. Then $\left(x^{*}, \lambda^{* 2}, \mu^{*}\right)$ can be seen as a saddle point to $\mathcal{L}_{g}$. It satisfies that $\mathcal{L}_{g}\left(x^{*}, \lambda, \mu\right) \leq$ $\mathcal{L}_{g}\left(x^{*}, \lambda^{*}, \mu^{*}\right) \leq \mathcal{L}_{g}\left(x, \lambda^{*}, \mu^{*}\right)$. These equalities hold when $\dot{\bar{V}}=0$, i.e.,

$$
\begin{aligned}
& \sum_{i \in \mathcal{N}}\left(\lambda_{i}^{2}-\lambda_{i}^{* 2}\right) g_{i}\left(z^{*}\right)+\left(\mu_{i}-\mu_{i}^{*}\right) h_{i}\left(z^{*}\right)=0 \\
& \sum_{i \in \mathcal{N}} f_{i}\left(z^{*}\right)=\sum_{i \in \mathcal{N}}\left\{f_{i}\left(x_{i}\right)+\lambda_{i}^{* 2} g_{i}\left(x_{i}\right)+\mu_{i}^{*} h_{i}\left(x_{i}\right)\right\} .
\end{aligned}
$$

Here, since $x_{i}$ is unchanged, it is clear from (9e) that $h_{i}\left(x_{i}\right)=$ 0 otherwise $\mu_{i}$ is unbounded, $\forall i \in \mathcal{N}$, then $\sum_{i \in \mathcal{N}} \mu_{i}^{*} h_{i}\left(x_{i}\right)=$ 0 . Therefore, if $\lambda_{i}^{* 2} g_{i}\left(x_{i}\right)=0, \forall i \in \mathcal{N}$, then we can conclude from $17 \mathrm{~b}$ that $x_{i}$ is the optimal solution. If $\lambda^{*} \neq 0$, then $\lambda_{i}>$ $0, \forall t>0$. This is because if $\lambda_{i}$ goes to zero, then $\bar{V} \rightarrow+\infty$ due to the term $-\frac{1}{2} \lambda_{i}^{* 2} \ln \lambda_{i}$ in $S_{i}^{g}$, which contradicts the fact that $\bar{V}$ is decreasing. We will reason by cases in the following.

1) If $g_{i}\left(z^{*}\right)<0$, then $\lambda_{i}^{*}=0, \lambda_{i}^{* 2} g_{i}\left(x_{i}\right)=0$ holds.

2) If $g_{i}\left(z^{*}\right)=0$, then $\lambda_{i}^{*}$ can be nonzero. Note that $x_{i}$ is a constant. Then $g_{i}\left(x_{i}\right) \leq 0$ or else $\lambda_{i}$ will be unbounded according to the dynamics $\dot{\lambda}_{i}=2 \lambda g_{i}\left(x_{i}\right)$.

a) If $g_{i}\left(x_{i}\right)=0, \lambda_{i}^{* 2} g_{i}\left(x_{i}\right)=0$ holds.

b) If $g_{i}\left(x_{i}\right)<0$, then $\lambda_{i}$ should be zero to render a stable equilibrium point, which contradicts the fact that $\lambda_{i}$ will not approach $0, \forall t>0$.

Therefore, $\lambda_{i}^{* 2} g_{i}\left(x_{i}\right)=0$ holds, and $\sum_{i \in \mathcal{N}} f_{i}\left(x_{i}\right)$ is the optimal value, which means that $x_{i}=x_{j}, \forall i, j$ is an optimal solution.

Remark 1. The LaSalle's invariance principle plays a crucial role, which allows the analysis in step 2 of the proof. Such an application of the LaSalle's invariance principle under delays is made valid thanks to the algorithmic dynamics (4) for the generalized Lagrangian that preserves smoothness and passivity. It should also be noted that the passivitybased phase lead compensation technique eliminates possible oscillations and ensures the convergence with cost functions not necessarily strictly convex.

\section{Application to Target Matching Problem}

Let us consider an environmental-monitoring problem that is formulated as a target matching problem [16].

$$
\begin{aligned}
& \min _{z_{l k} \geq 0} \sum_{l=1}^{N} \sum_{k=1}^{M} z_{l k}\left\|w_{l}-q_{k}\right\| \\
& \text { s.t. } \sum_{l=1}^{N} z_{l k}=1, k=1, \ldots, M, \quad \sum_{k=1}^{M} z_{l k}=1, l=1, \ldots, N
\end{aligned}
$$

where $z=\operatorname{col}\left(z_{1}, \ldots, z_{N}\right) \in \mathbb{R}^{N M}$ is the decision variable with $z_{l}=\operatorname{col}\left(z_{l 1}, \ldots, z_{l M}\right) \in \mathbb{R}^{M}$, and $N, M$ are the number of Robots and Targets, respectively. The variable $z_{l k} \in \mathbb{R}$ 
denotes the matching label for Robot $l$ and Target $k$. The term $\left\|w_{l}-q_{k}\right\|$ denotes the distance from Robot $l$ to Target $k$, which is regarded as a constant (given by sensing). Note that the linear programming problem (18) with continuous variables is a strictly relaxation from integer programming. The optimal solution for $z_{l k}$ is either 1 or 0 , which implies the matching status between Robot $l$ and Target $k$ [16]. We reformulate (18) as a consensus-based distributed optimization problem and use notation $x_{i(l k)}$ to denote the estimation of $z_{l k}$ from agent $i$.

Consider an area of $100 \times 100\left[\mathrm{~m}^{2}\right]$ with $N=5$ Robots and $M=5$ Targets. The positions of Robots and Targets are shown in Figure 2(a)

The communication graph is set to be a ring graph with $a_{i j}=4, \forall(i, j) \in \mathcal{E}$, the other parameters are defined as $m=2, b_{2}^{i}=5, c_{1}^{i}=1, c_{2}^{i}=10, \eta=1$, the initial condition is $\left.\left(x_{i}, \xi_{i}, \lambda_{i}, \mu_{i}, \rho^{i}\right)\right|_{t=0}=(\mathbf{0}, \mathbf{0}, 0.01,0, \mathbf{0})$ and the stepsize is set to 0.001 in Simulink. The trajectories of of $x_{i}, i=1, \ldots, 5$ in algorithm (9) without communication delay converge to the optimal solution, as shown in Figure 3(a), which validates Theorem 1

Next, we assume that there exist unknown and heterogeneous constant delays between any two neighboring agents. The communication delays can bring in instability for algorithm (9) (see, e.g., [3]). Thus, let us adopt algorithm (11) with scattering transformation (14) to enhance robustness against delays, and consider heterogeneous communication delays $T_{i j} \in[0.2,0.3][\mathrm{s}], \forall(i, j) \in \mathcal{E}$. The trajectories of $x_{i}$, $i=1, \ldots, 5$ converge to the optimal solution as shown in Figure 3(b), validating Theorem 2 Note that the agents' states diverge for the present delays in the absence of the scattering transformation. Therefore, the scattering transformation serves as a key technique for delay robustification. The corresponding results are shown in Figure 2(b), illustrating the matching between Robots and Targets.

\section{CONCLUSION}

In this work, we have addressed the distributed constrained optimization problem under inter-agent communication delays from the perspective of passivity. A smooth continuous-time algorithm for distributed constrained optimization with general convex objective functions has been proposed. The scattering transformation has been incorporated into the proposed

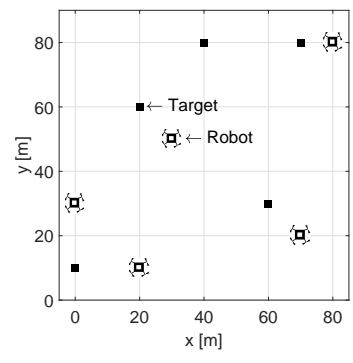

(a) Robots and Targets positions.

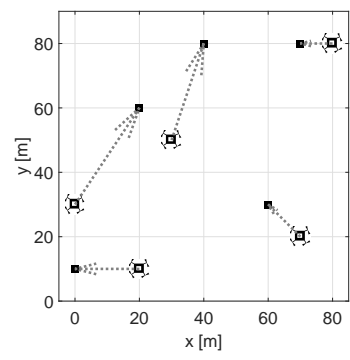

(b) Matching results.
Fig. 2. Robots and Targets in the $100 \times 100\left[\mathrm{~m}^{2}\right]$ area.
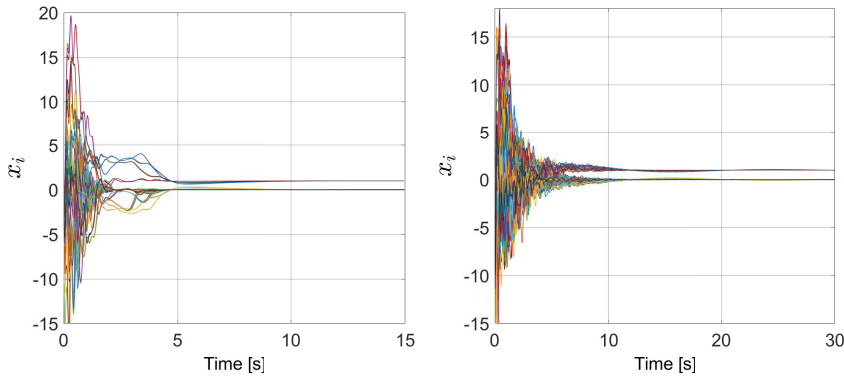

(a) The algorithm 9 without commu- (b) The algorithm (11) with scatternication delay. ing transformation under heterogeneous communication delays.

Fig. 3. The trajectories of $x_{i}, i=1, \ldots, 5$.

algorithm to enhance the robustness against unknown and heterogeneous communication delays.

\section{REFERENCES}

[1] J. Wang and N. Elia, "A control perspective for centralized and distributed convex optimization," in 50th IEEE conference on decision and control and European control conference, 2011, pp. 3800-3805.

[2] T. Yang, X. Yi, J. Wu, Y. Yuan, D. Wu, Z. Meng, Y. Hong, H. Wang, Z. Lin, and K. H. Johansson, "A survey of distributed optimization," Annual Reviews in Control, 2019.

[3] T. Hatanaka, N. Chopra, T. Ishizaki, and N. Li, "Passivity-based distributed optimization with communication delays using PI consensus algorithm," IEEE Transactions on Automatic Control, vol. 63, no. 12, pp. 4421-4428, 2018

[4] T. Miyano, S. Yamashita, T. Hatanaka, K. Shibata, T. Jimbo, and M. Fujita, "Continuous-time optimization dynamics mirroring ADMM architecture and passivity-based robustification against delays," IEEE Transactions on Control of Network Systems, 2020, early access.

[5] S. Yamashita, T. Hatanaka, J. Yamauchi, and M. Fujita, "Passivitybased generalization of primal-dual dynamics for non-strictly convex cost functions," Automatica, vol. 112, p. 108712, 2020.

[6] M. Li, G. Chesi, and Y. Hong, "Input-feedforward-passivity-based distributed optimization over jointly connected balanced digraphs," arXiv preprint arXiv:1905.03468, 2019.

[7] L. Su, M. Li, V. Gupta, and G. Chesi, "Distributed resource allocation over time-varying balanced digraphs with discrete-time communication," arXiv preprint arXiv:1907.13003, 2019.

[8] T. Hatanaka, N. Chopra, M. Fujita, and M. W. Spong, Passivity-based control and estimation in networked robotics. Springer, 2015.

[9] S. Yang, Q. Liu, and J. Wang, "Distributed optimization based on a multiagent system in the presence of communication delays," IEEE Transactions on Systems, Man, and Cybernetics: Systems, vol. 47, no. 5, pp. 717-728, 2016.

[10] A. Cherukuri, E. Mallada, and J. Cortés, "Asymptotic convergence of constrained primal-dual dynamics," Systems \& Control Letters, vol. 87, pp. 10-15, 2016.

[11] M. Li, "Generalized Lagrange multiplier method and KKT conditions with an application to distributed optimization," IEEE Transactions on Circuits and Systems II: Express Briefs, vol. 66, no. 2, pp. 252-256, 2019.

[12] T. Holding and I. Lestas, "On the emergence of oscillations in distributed resource allocation," Automatica, vol. 85, pp. 22-33, 2017.

[13] D. Feijer and F. Paganini, "Stability of primal-dual gradient dynamics and applications to network optimization," Automatica, vol. 46, no. 12, pp. 1974-1981, 2010.

[14] R. J. Anderson and M. W. Spong, "Bilateral control of teleoperators with time delay," IEEE Transactions on Automatic control, vol. 34, no. 5, pp. 494-501, 1989.

[15] H. L. Smith, An introduction to delay differential equations with applications to the life sciences. Springer New York, 2011, vol. 57.

[16] T. Miyano, S. Yamashita, T. Hatanaka, K. Shibata, T. Jimbo, and M. Fujita, "Design of continuous-time ADMM and convergence analysis based on passivity," Transactions of the Society of Instrument and Control Engineers, vol. 55, pp. 286-293, 2019, (in Japanese). 